\title{
Utilization of Huge Written Text Corpora for Conversational Speech Recognition
}

\author{
Xinhui $\mathrm{Hu}^{1,2}$, Hirofumi Yamamoto ${ }^{1}$, Jinsong Zhang ${ }^{1,2^{*}}$, Keiji Yasuda $^{1,2}$, \\ Youzheng $\mathrm{Wu}^{1,2}$, Hideki Kashioka ${ }^{1,2}$ \\ ${ }^{1}$ National Institute of Information and Communications Technology, \\ ${ }^{2}$ ATR Spoken Language Communication Group, \\ Hikaridai 2-2-2, Seika-cho, Soraku-gun, Kyoto 619-0228, Japan \\ \{xinhui.hu, hirofumi.yamamoto, jinsong.zhang, keiji.yasuda, youzheng.wu, hideki.kashioka\}atr.jp
}

\begin{abstract}
In this paper, we propose a new sentence selection method using large written text corpora to augment the language model of conversational speech recognition in order to resolve the insufficiency of in-domain training data coverage in conversational speech recognition. In the proposed method, the large written text corpora are clustered by an entropybased method. Clusters similar to the target development set are selected automatically. Next, utterances are selected and mixed with the original conversational training corpus, and language models for conversational speech recognition are built. In our experiments, a different speech style test set that is not covered by original conversational training data is used for evaluation. The perplexity of the test set was reduced from 249.6 to 210.8 , and the word recognition accuracy was improved by approximately $5 \%$ by using our method.
\end{abstract}

Index Terms: data collection, training data coverage, language model, conversational speech recognition.

\section{Introduction}

The language model (LM) plays an important role in many applications, including speech recognition. It is generally trained by using an annotated corpus. The annotated corpus is required to be sufficiently large to avoid data sparseness. Moreover, it should be in the same style as one's task, that is, the corpus should be close to the domain in both content and expression style.

For any corpus, there exist two common problems. The first problem is that it is very time-consuming and expensive to enlarge the corpus. The second problem is that it is difficult to cover a wide range of domains. In tasks involving conversational speech, the ideal training material, i.e., transcripts of conversational speech, is costly to produce, and therefore, it limits the amount of available training data.

To overcome the above problems, considerable research has been conducted on automatically adding data from outside the existing annotated corpus. With the rapid expansion of the Internet, data, not only in huge amounts but also in a variety of forms, are available from websites [1,2]. Adaptation data are generally obtained by submitting words from initial hypotheses of user utterances as queries to a Web search engine. However, these queries treat words as individual tokens and ignore function words. Such a search strategy typically generates text with a nonconversational style, which is not ideally suitable for automatic speech recognition. In [3], instead of downloading the actual web pages, N-gram counts are directly retrieved by the search engine. This approach generates valuable statistics for the set of $\mathrm{N}$-grams to the ones occurring in the baseline model. In all these methods, the estimation of the probabilities for the tokens being searched is improved; however, the coverage of the search is limited.
In this paper, we propose a novel method for extracting sentences from a large general corpus. Although there exist many difficulties in sharing these general texts due to different domain, it is possible to collect useful data from these huge data. In fact, there are many sentences originated from speech, such as news broadcasts, dialogue quotations. Instead of using a limited number of $\mathrm{N}$-gram tokens derived from the training corpus, we use sentence entropy to compute the similarity between searched data and target data. The preservation of the domain and style is ensured by using entire sentences and not just a small portion of the N-gram, and the tokens appear only in the training corpus. The purpose of this research is to augment a language model for Chinese conversational speech recognition whose domain is travel dialogs. In Section 2, after presenting our considerations on selecting a large general text corpus as our data resource, we present the entropy-based sentence clustering approach for character-based utterances in Chinese. In Section 3, we describe the word segmentation method for clustered utterances, and the building of the language model by combining selected data and the baseline corpus. We report our experiments in speech recognition using the augmented language model. In section 4 , we provide a summary of this study.

\section{Data Collection by Sentence Clustering}

\subsection{Considerations in Selecting Source Data}

When compared to English and Japanese languages, the Chinese language has special problems with regard to the construction of corpora for natural language processing. Excluding mainland China, where the GB code is officially used, some other coding characters exist in other regions, such as the BIG5 code used in Taiwan and Hong Kong. The variety of character coding causes difficulties when sharing language resources. Another common difficulty with the Chinese language is the problem of word segmentation. Unlike English, there is no blank space to explicitly separate words; it is generally a prerequisite step to segment a Chinese utterance into a word sequence before performing any language processing. Due to the ambiguities in word definition, the segmentation continues to remain a difficult task.

In ATR, a multilanguage speech translation system that performs Japanese-English and Japanese-Chinese translations has been developed. In the Japanese-English translation system, a bilingual $1000 \mathrm{~K}$ utterance text corpus is used. In the Japanese-Chinese translation system, a subset of $500 \mathrm{~K}$ utterances is adopted from the $1000 \mathrm{~K}$ utterances for building language models for translation and speech recognition [4]. Chinese data are translated mainly from Japanese data, but and some data are translated from English.

* Present in Beijing Language and Cultural University 
There are some special problems related to the Chinese corpus used in the ATR speech translation system. Firstly, the data quantity is insufficient; it is only half the quantity in the other two languages. Secondly, all the Chinese text data are translated from other languages, mainly from Japanese and a few from English. There is some bias in the expressions (many sentences do not read like natural utterances from a native speaker), and the text data is short of Chinese proper nouns.

We obtain a set of Chinese text from the LDC corpora as the source data from which we select the required sentences. Most of the texts are nonconversational, but there is a fair amount of chat-like material that is similar to conversational speech (though disfluencies are often omitted). As this data set is obtained from broadcast news, it is considered to be suitable for reading and is relatively closer to the conversational style than the other texts such as newspaper texts. LDC data has been formally annotated with adequate tags in, for example, XML format. It is easy to extract the necessary information from this markup. We need not prepare queries for search based on $\mathrm{N}$-gram tokens from the existing corpus. Our other aim was to extract utterances containing native-like expressions and Chinese proper nouns. For collecting such types of proper nouns, the newswire is considered to be more effective and more reliable than the texts gathered from the Internet.

\subsection{System Configuration}

The proposed method of extracting sentences is based on the following considerations. Entire sentences are divided into several subsets (clusters) by clustering; each subset (cluster) is assumed to contain sentences with familiar characteristics. Each cluster is then evaluated by computing the perplexity of a development data set. The clusters with low perplexities are considered to be greatly similar to the development data set, and hence, they are finally selected. The selection procedure is divided into two steps. These steps are described in Figure 1 .

In the first step, all the LDC texts are clustered into $\boldsymbol{m}$ (here, $\boldsymbol{m}$ is 10) groups (we consider them as the big group, and term them "BigClus"), and the cluster with the minimum perplexity is selected as the output.

In the second step, the cluster selected in the first step is clustered again in the same way. $\boldsymbol{m}$ subgroups are obtained (we consider them as the little group, and term them "LitClus") and ranked on the basis of their perplexities. The final selection is made from these subgroups.

The reason for clustering twice is that the difference between the conversational texts and the news text is large, so more rigorous limitation is required. In our experiments, the processing time is also found to be shorter than that for single clustering with a large cluster number $m \times m$.

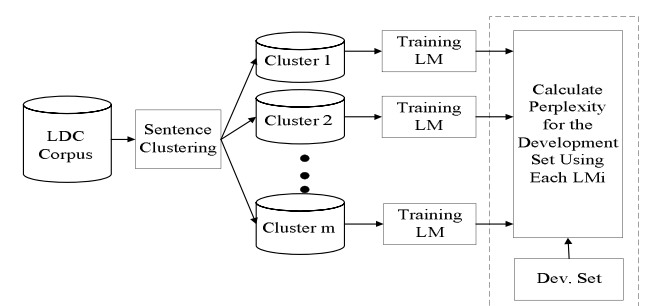

Figure 1. Flow of Sentence Clustering in a Single Step

\subsection{Algorithm of Sentence Clustering}

Sentence clustering is conducted by minimizing the total entropy of all the sentences with respect to their corresponding language models. The procedure is described as follows:

(1) As an initial state, the temporary cluster number is assigned to a specified model order (in this case, 10). The unit of clustering is sentence, the initial sentences is selected in span of this order from the whole corpus. This processing can provide a stable initial state for clustering.

(2) N-gram models are calculated for each cluster (here, a unigram is used.)

(3) Looping begins according to the number of loops specified in the option (in this case, 30 ).

(3.1) A text is extracted from a file list.

For clusters that contain the extracted text, we remove the texts from those clusters, and the change in entropy due to the removal is calculated. For all the other clusters, the change in entropy when the texts are added is calculated. On the basis of these changes, we perform a search for the destination cluster that results in an overall decrease in the total entropy. The utterances are then moved from the temporary clusters to the optimal destination cluster.

(3.2) Return to step (3.1) until no more texts can be processed.

(4) Stop if there is no change in total entropy. If there is a change, go to step (3).

\subsection{Data for Sentence Clustering}

Table 1 lists the data used in sentence clustering and evaluation. Both the development set (Dev. Set) and test set (Test Set) are selected from the ATR annotated corpus. The development set is used to evaluate each language model built with the selected clusters (as shown in Figure 1). The test data are used for the selection of the clustered data that have been evaluated and ranked by the development set.

\begin{tabular}{|l|c|c|}
\multicolumn{1}{c|}{ Table 1. Data Used for Sentence Clustering } \\
\begin{tabular}{|l|c|c|}
\hline Data Type & $\begin{array}{c}\text { Size } \\
\text { (Char) }\end{array}$ & Description \\
\hline Large Corpus & $97.3 \mathrm{M}$ & $\begin{array}{c}\text { Chinese text corpus from } \\
\text { LDC, LDC95T13 } \\
\text { Dev. Set }\end{array}$ \\
Test Set & $33.7 \mathrm{~K}$ & $\begin{array}{c}\text { ATR annotated corpus } \\
\text { Avaluation data set for ATR } \\
\text { speech translation system }\end{array}$ \\
\hline
\end{tabular}
\end{tabular}

\subsection{Text Normalization and Character-based} Utterances

The original LDC texts are labeled with XML tags. The basic unit of the text is a paragraph, which often contains several sentences. Since we aim to extract sentences from this data, we conduct the following normalization for the original LDC texts.

(1) Remove XML tags from the original LDC texts and only extract text paragraphs.

(2) Divide paragraphs into segments according to punctuation (e.g., period, question mark, and exclamation mark). A comma may also be used to delimit a segment when it is too long (longer than 100 Chinese characters). 


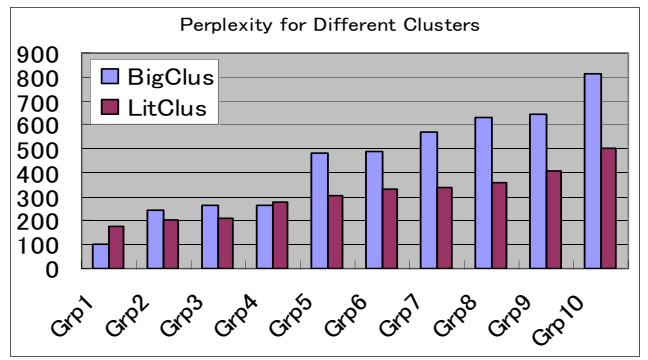

Figure 2. Perplexity of Clustered Groups

(3) Convert all characters into GB2312 codes. The sentences that fail this conversion are discarded.

After the normalization, a total of $4.14 \mathrm{M}$ utterances were obtained. We adopt character-based utterances for clustering since we consider that any sentence is ultimately composed of characters and that different topics or styles contain different characters and their combinations. These character-based utterances are obtained using the following step.

(4) Separate each character by an ASCII space for each utterance.

A unigram model is adopted for the sentence clustering of Figure 1; however, trigram models are adopted for other language models.

Figure 2 shows the perplexities of the development set for each LM built by each cluster in these two clustering steps; it is ordered by their perplexities. BigClus refers to the results of all the $4.14 \mathrm{M}$ utterances, and LitClus refers to the results of the optimal group (Grp1) in the first step. The rank of group reflects similarity to the target data (spoken data). The littler of perplexity, the more similar the group to the target.

\subsection{Selection of Clustered Data}

We accumulate the 10 clusters of the above little group (LitClus), build the language model (trigram), and observe the perplexity of the test set of these models. Figure 3 shows these perplexities. The $\mathrm{x}$-coordinate indicates the number of clusters. The number 0.1 denotes that only Grpl is added, 0.2 denotes that Grp2 is added, and so on.

For the purpose of comparison, we also provide the perplexities of the accumulated clusters of the big group (BigClus), the perplexities with the accumulations of randomly selected data from the entire LDC texts, and the perplexities with the accumulations of randomly selected data from the entire little group (LitClus). We can observe from the figure that the perplexities arising from the clustered data are smaller than those derived from randomly selected ones. When all the data are used, their perplexities are similar. In the case of the randomly selected data, the perplexity decreases with an increase in the number of data groups. However, for the clustered data of LitClus, their perplexities decrease initially; however, when the data size exceeds a certain threshold, the perplexity increases. The threshold value here is 0.4 ; in other words, the optimal size is 4 (clusters Grp 1-4 are used together). The data accumulation is conducted in the order of group's perplexity. As mentioned before, the group with big perplexity is regarded as having more unnecessary data, so the curve of the perplexity appears as "V" shape. In the above evaluation, the lexicon is automatically produced by the accumulated data. So it is not necessary to handle the lexicon and training data. These procedures lead us to the following conclusion,



Figure 3. Perplexity of Test Set with Data Accumulation .

(1) Sentences obtained from clustered data are more effective than those derived from randomly selected data.

(2) The little group of clustered data is better than the big group. Although the difference diminishes as the amount of training data increases, there is an optimal point for the little clustered groups. This signifies that only a small subset of the clustered data is required to obtain the minimum perplexity, and therefore, we can achieve a better result as compared to when the entire data are utilized.

In accordance with the above, we finally extracted $112 \mathrm{~K}$ utterances from the LDC texts.

\section{Word Segmentation and Addition of Clustered Data to Training Corpus}

\subsection{Word Segmentation}

The selected sentences need to undergo word segmentation before being added to the training corpus. To avoid the generation of unknown words, which are generally composed of fragmented words and characters, a word segmenter is built with a large database. This database contains the following items:

(1) Several annotated corpora, including ATR's annotated conversational corpus, People's Daily corpus built by the Peking University, and training data for Chinese segmentation provided by Sighan [5]. The development of the ATR Chinese corpus is mainly based on the People's Daily's specification.

(2) Several dictionaries. They include one dictionary obtained from websites and one dictionary used in China's high-technology development program. These vocabularies total $200 \mathrm{~K}$ words.

(3) A table of Chinese proper nouns containing location names, organization names, famous Chinese sightseeing locations, Chinese food menus, and names of people collected from the Beijing area. Some sport names are also included.

Using these large-scale resources, we built an HMM-based word segmenter and used it to segment the clustered sentences.

Table 2 shows the details of the final data for training the language model. A total of $12.7 \mathrm{~K}$ new lexicon words are collected from the clustered sentences.

Table 2. Final Corpus for Training Language Model

\begin{tabular}{|l|c|c|c|}
\hline \multicolumn{1}{|c|}{ Data Set } & Utt. & Total Words & Lexicon Size \\
\hline ATR Baseline & $417 \mathrm{~K}$ & $3.45 \mathrm{M}$ & $42.3 \mathrm{~K}$ \\
Clustered & $112 \mathrm{~K}$ & $2.15 \mathrm{M}$ & $31.6 \mathrm{~K}$ \\
Combination & $529 \mathrm{~K}$ & $5.60 \mathrm{M}$ & $55.0 \mathrm{~K}$ \\
\hline
\end{tabular}




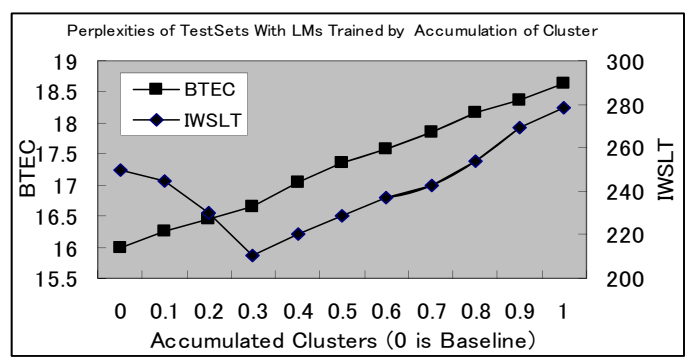

Figure 4. Perplexities of the Baseline and Accumulation of Clustered Data

\subsection{Adding Clustered Sentences to Training Corpus}

In order to investigate what happens to the language model after the selected sentences are added to the training data (ATR corpus), we first train a baseline model with the existing training corpus and then add the clustered sentences (LitClus groups) to the training corpus. Ten models were built. The perplexities of each language model with respect to two test sets are computed. One of the test sets is the same as that used in the above evaluations (denoted as BTEC), and the other is a test data used in IWSLT2006 (denoted as IWSLT) for which the expression style is very different from the ATR corpus in terms of vocabulary, utterance length, etc. These perplexities are shown in Figure 4. The details of these two data sets are shown in Table 3, which is discussed later for the evaluation of speech recognition. Here, $\mathrm{x}$-coordinate $=0$ refers to the baseline corpus, 0.1 indicates that group 1 of LitClus is added, 0.2 indicates that group 2 is added, and so on. The perplexity $\mathrm{w}$

ith the BTEC was found to linearly increase with the accumulation of clusters. This may be explained by the fact that the domain or expression style of the clustered data is somewhat different from the original conversational corpus. However, the perplexity with the IWSLT decreases at the beginning of accumulations, but starts increasing after some accumulations (at approximately $x$-coordinate $=0.4$ ). This also means that the IWSLT is greatly different from the baseline corpus, and this new selected data is useful to improve its performance. We select 0.4 as the optimal point for the cluster accumulation. In this range, the perplexity with the BTEC increases from 16.0 to 17.0 , i.e., by approximately $6.3 \%$. If all the clustered sentences are added, the increment is 2.6 , i.e., approximately $15.0 \%$. This is illustrated by a speech recognition experiment presented in the next section.

\subsection{Experiments on Speech Recognition with the Augmented Language Model}

Perplexity is an important criterion that is used to evaluate language models; however, it is not the only one. Our goal is to improve the language model for conversational speech recognition in the travel domain. To confirm the validity of the addition of clustered sentences in speech recognition, we build a bigram language model and a trigram language model for decoding and rescoring in speech recognition, respectively. Two sets of test data with different expression styles, which have been used and shown in Figure 4, are used for the evaluation of recognition. The word accuracy of speech recognition, perplexity of trigram model with respect to these two test sets, and additional information on the two data sets are presented in Table 3. For comparisons, the perplexities and word accuracies of the previous experiments (in which only the ATR corpus was used) are shown in Table 3 (on the Table 3. Performance Changes by Addition of Training Data.

\begin{tabular}{|c|c|c|}
\hline DataSet & BTEC & IWSLT \\
\hline Utterances & 510 & 500 \\
Words & $2.8 \mathrm{~K}$ & $5.5 \mathrm{~K}$ \\
Perpw3.(New/Old) & $33.7 / 33.3$ & $210.8 / 249.6$ \\
Wd.Acc(New/Old) & $80.9 \% / 79.1 \%$ & $46.6 \% / 41.7 \%$ \\
\hline
\end{tabular}

right-hand side of the solidus). From this table, it can be observed that the word accuracy of speech recognition has been improved by the addition of training data from the clustered data of both test sets, especially for the IWSLT test set. When using IWSLT data, we observe a substantial decrease in the perplexity, while with BTEC data, we observe a small increase in the perplexity.

\section{Conclusions}

In this paper, we have described an entropy-based sentence clustering method for selecting sentences from a large text corpus, and used them as additional training data for a language model for Chinese conversational speech recognition. We have shown that it is possible to extract a portion of data from the text corpus with different domain. For our speech recognition in travel domain, it is necessary to cluster the original text twice.

Using above mentioned method, we obtained more than 100 $\mathrm{K}$ utterances. With the newly added lexicon items and corresponding sentences, the coverage of the combined corpus has been increased. With regard to the practical application of this approach to actual speech recognition, we have demonstrated a clear improvement in the word error rate. In future studies, we will utilize much more information on the target corpus during the selection process. For example, stricter restrictions can be placed on the utterance length in the initial steps of normalizing texts. We will also make comparison with other methods of data collection, such as by web searching. Further, we plan to explore the language model combination in which the baseline model and supplemental model are trained independently so that only the latter needs to be re-instantiated by using our proposed approach, if necessary.

\section{Acknowledgments}

This study was partly supported by a Grant-in-Aid for Scientific Research on Priority Areas in Japan as a part of Cyber Infrastructure for the Information Explosion Era (under Grant No. 19024074).

\section{References}

[1] T. Ng, M. Ostendofr, M.Y. Hwang, M. Sui, I. Bulyko, X. Lei, "Web-Data Augmented Language Models for Mandarin Conversational Speech Recognition”, In Proc. ICASSP, 2005, pp.589-592.

[2] I. Bulyko, M. Ostendorf, A. Stolcke, "Getting More Mileage from Web Text Sources for Conversational Speech Language Modeling Using Class-dependent Mixtures", in Proc. HLT/NAACL, 2003, pp.7-9.

[3] X. Zhu and R. Rosenfeld, "Improving Tragram Language Modeling with the World Wide Web", in Proc. ICASSP, 2001, pp.I533-536.

[4] G. Kikui, E. Sumita, T. Takezawa, S. Yamamoto, "Creating Corpora for Speech-to-speech Translation", In Proc. Eurospeech, 2003, pp.381-384.

[5] http://www.sighan.org 